\title{
Sensitivity of 4D-STEM to Valence Electron Distribution Based on Multipole Density Formalism
}

\author{
Lijun Wu, Qingping Meng and Yimei Zhu \\ Brookhaven National Laboratory, Upton, New York, United States
}

Valence electrons are electrons involved in chemical bonding that determine a material's functionality. The ability to accurately measure valence electron distribution is at the heart of condensed matter and materials science research. Measurement of charge density is traditionally performed using single-crystal x-ray diffraction (SCXRD), or combination of SCXRD and quantitative convergent-beam electron diffraction (QCBED), which usually requires high quality large single crystals [1]. Recent advancement in detector technology opened a door for 4D-STEM, which yields a diffraction pattern for each scanning position. It has been demonstrated that 4D-STEM can be used to measure the charge density of 2D materials with atomic resolution [2]. However, the measurement is based on center-of-mass (COM) and only valid for extremely thin samples. Moreover, it only measures the projected total charge density rather than the electrons that bond the atoms together. Here, we demonstrate that the 4D-STEM is sensitive to the valence electron distribution based on simulations, thus can be used to map aspherical valence-electron distribution in quantum materials. The electron density of a crystal can be modeled based on atom-centered multipole density functions (MDF) with the electron density of the atom as [3]:

$\rho_{\text {atom }}(r, \theta, \varphi)=P_{c} \rho_{\text {core }}(r)+P_{v} \kappa^{3} \rho_{\text {val }}(\kappa r)+\sum_{L} \kappa^{\prime 3} R_{L}\left(\kappa^{\prime} r\right) \sum_{M} P_{L M \pm} d_{L M \pm}(\theta, \varphi)$

Here $\rho_{\text {core }}$ and $\rho_{v a l}$ are the spherical atomic core and spherical valence electron density, $P_{c}$ and $P_{v}$ are the electron population of core and valence component, $\kappa$ is a parameter describing expansion $(\kappa<1)$ or contraction $(k>1)$ of the valence shell. The third term is the multipole contribution, representing aspherical distribution of valence electrons. $R_{L}(r)$ is the radial function. $\theta$ and $\varphi$ are the angular coordinates of $\boldsymbol{r}$. Our interest is in the second and third terms characterizing the distribution of bonding electrons, which is a small fraction of the total electron density but determines material's behavior. Based on above formula, the corresponding structure factors can be calculated and used to simulate 4D-STEM patterns through either Bloch wave method or multislice method. Fig. 1(a) shows a set of 4D-STEM patterns of 2H-MoS 2 calculated using Bloch wave method based on IAM (Independent Atom Model with spherical neutral atoms) model. Each disk corresponds to the central part (0-23 mrad) of a 4D-STEM pattern at a scan position. In 4D-STEM, the reflection disks heavily overlap, and form interference patterns that change with probe positions. For a typical convergent semi-angle of 20-30 mrad, the central part of the disk, e.g. 0-30 mrad, has the best signal and carries valence electron information. $2 \mathrm{H}-\mathrm{MoS}_{2}$ has $\mathrm{P}_{3} / \mathrm{mmc}$ space group with Mo at $-6 \mathrm{~m} 2$ and $\mathrm{S}$ at $3 \mathrm{~m}$ site. Mo atom has five $4 \mathrm{~d}$ and one $5 \mathrm{~s}$ valence electrons, while $\mathrm{S}$ has two $3 \mathrm{~s}$ and four $3 \mathrm{p}$ valence electrons. We define the MDF model with $\mathrm{Mo}^{4+}$ (two $4 \mathrm{~d}$ electrons) octupole $\mathrm{P}_{33+}=1$ and $\mathrm{S}^{2-}$ (two $3 \mathrm{~s}$ and six $3 \mathrm{p}$ electrons) octupole $\mathrm{P}_{33+}=0.5$ and hexadecapole $\mathrm{P}_{43+}=0.5$. The corresponding valence electron distribution of (001) in the Mo and S plane is shown in Fig. 2(a,b). Fig. 1(b-g) compare 4D-STEM patterns between IAM and MDF at thickness of $\mathrm{t}=10 \mathrm{~nm}$ and $\mathrm{t}=20 \mathrm{~nm}$. It is seen that the 4D-STEM patterns change noticeablly at $\mathrm{t}=10 \mathrm{~nm}$, and become significant at $\mathrm{t}=20 \mathrm{~nm}$, indicating a high sensitivity to the valence electron distribution for relatively thick samples. We therefore propose to map valence electron distribution by refining 4D-STEM patterns using MDF. For comparison, we also compare the COM and charge density obtained from 4D-STEM calculated at very thin sample $(\mathrm{t}=1.23 \mathrm{~nm})$, as shown in Fig. 2(c-f). Although we can see small change in charge density, we are not able to reveal the valence electron distribution [4]. 


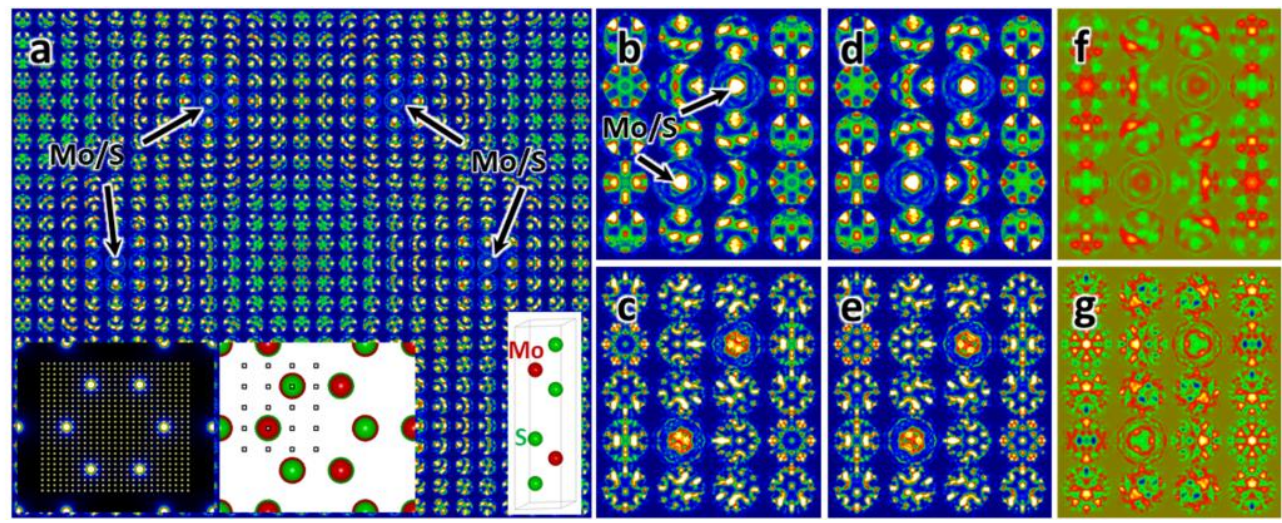

Figure 1. Figure 1. (a) A set of 4D-STEM patterns consisting of the central part $(0-23 \mathrm{mrad})$ of the individual CBED pattern at scan positions marked by white dots in the bottom-left inset. The calculations are performed by Bloch wave method with IAM model for electron beam energy=200 $\mathrm{KeV}$ and thickness $=10 \mathrm{~nm}$. The convergent semi-angle is $21 \mathrm{mrad}$. Note, the CBED patterns preserve the site symmetry. The insets in the bottom left and middle are the [001] projection of the total electron density and MoS2 structure model, respectively. The inset in the bottom-right is the 3D view. (b-e) 4D-STEM patterns consisting of 20 4D-STEM patterns around two Mo/S sites calculated based on (b,c) IAM and $(\mathrm{d}, \mathrm{e}) \mathrm{MDF}$ models for sample thickness of $(\mathrm{b}, \mathrm{d}) 10 \mathrm{~nm}$ and $(\mathrm{c}, \mathrm{e}) 20 \mathrm{~nm}$. The scan positions are marked by the black squares in the bottom-middle inset in (a). (f,g) Intensity difference between (b) and (d), and (c) and (e).

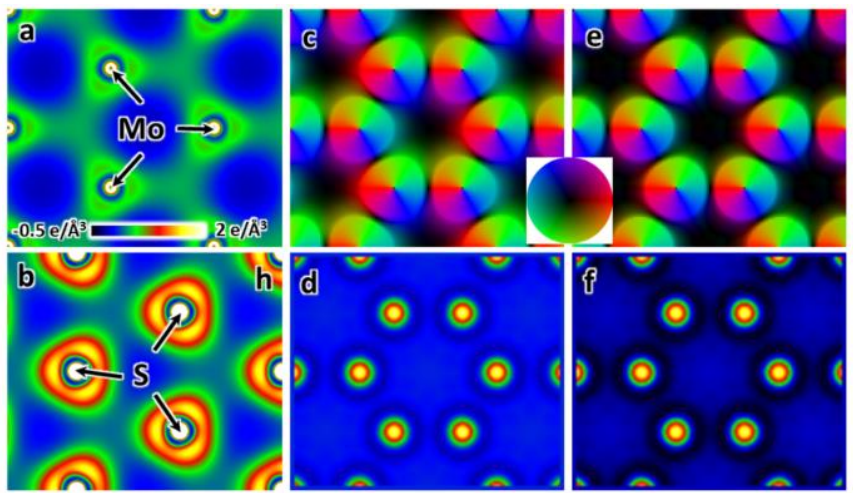

Figure 2. Figure 2. (a,b) Valence electron distribution of (001) plane at (a) Mo plane and (b) S plane calculated based on a MDF model with Mo4+ octupole P33+=1, S2- octupole P33+=0.5 and hexadecapole $\mathrm{P} 43+=0.5$. (c,e) Center of mass of 4D-STEM patterns calculated based on (c) IAM and (e) MDF at thickness $\mathrm{t}=1.23 \mathrm{~nm}$ (1 unit cell thick). The inset shows the vector color wheel. (d,f) [001] projected total charge density calculated by taking divergence from (c) and (e), $\rho \propto-\nabla \cdot \operatorname{COM}$.

\section{References}

[1] Y. Zhu, J. C. Zheng, L. Wu, A. I. Frenkel, J. Hanson, P. Northrup, W. Ku, Phys. Rev. Lett. 99 (2007), 037602.

[2] W. Gao et al. Nature 575 (2019), 480-484.

[3] P. Coppens, X-Ray Charge Densities and Chemical Bonding (Oxford University Press, New York, 1997).

[4] The authors acknowledge funding from the US DOE-BES, Materials Science and Engineering Division, under Contract No. DESC0012704. 